\section{International Scientific Journal Theoretical \& Applied Science}

p-ISSN: 2308-4944 (print) e-ISSN: 2409-0085 (online)

Year: $2016 \quad$ Issue: 5 Volume: 37

Published: $30.05 .2016 \quad \underline{\text { http://T-Science.org }}$

SECTION 27. Transport.
Andrey Stanislavovich Reshenkin professor, candidate of technical sciences, head of department

Don State Technical University, Russia v.serge.79@mail.ru

Sergey Sergeevich Vorobyev assistant professor, candidate of technical sciences Don State Technical University, Russia

Victor Gennad'evich Babkin student of Don State Technical University, Russia

Andrey Yur'evich Ganchurin student of Don State Technical University, Russia

Tokhir Kamilzhonovich Sattarov student of Don State Technical University, Russia

\title{
SYSTEM OF MODELS FOR THE COMPREHENSIVE ASSESSMENT OF THE CONDITION OF THE CAR SERVICE ENTERPRISES
}

Abstract: One of the urgent problems having important theoretical and practical significance is the development and implementation of the region transport control in which the models for the assessment of organizations and enterprises including car service centers are the most important element.

The article shows the possibility of using models of different level to assess the state of industrial enterprises, service organizations to determine the condition of service centers.

Key words: system of models, assessment of the condition, car service enterprises.

Language: Russian

Citation: Reshenkin AS, Vorobyev SS, Babkin VG, Ganchurin AY, Sattarov TK (2016) SYSTEM OF MODELS FOR THE COMPREHENSIVE ASSESSMENT OF THE CONDITION OF THE CAR SERVICE ENTERPRISES. ISJ Theoretical \& Applied Science, 05 (37): 16-21.

Soi: http://s-o-i.org/1.1/TAS-05-37-3 Doi: crossef http://dx.doi.org/10.15863/TAS.2016.05.37.3

\section{СИСТЕМА МОДЕЛЕЙ ДЛЯ КОМПЛЕКСНОЙ ОЦЕНКИ СОСТОЯНИЯ ПРЕДПРИЯТИЙ АВТОСЕРВИСА}

Аннотация: Одной из актуальных проблем, имеющих важное теоретическое и практическое значение, является разработка и внедрение системы управления автотранспортом региона, в которой важнейшим элементом являются модели для оценки состояния организаций, предприятий, в том числе и автосервиса.

В статье показана возможность использования моделей различного уровня, для оценки состояния промышленных предприятий, организаций сервиса, для определения состояния предприятий автосервиса.

Ключевые слова: система моделей, оченка состояния, предприятия автосервиса.

При построении моделей для отдельных (предприятий автосервиса) ПАС с незначительно изменяющейся во времени структурой и отдельных предприятий в условиях глубоких внутренних структурных изменений используются три подхода [1-3]:

-подход на основе расчета финансовоэкономических параметров, где основным является прибыль или индекс прибыли;

-системный подход, учитывающий взаимодействие производственных процессов, инновационных технологий с финансовоэкономическими структурами предприятия; -подход на основе построения межпродуктового баланса.

Для построения модели любого ПАС на основе выделенных подходов необходимо задать виды выпускаемой им продукции (услуги).

Для каждого вида продукции вводятся различные уровни $(У)$, характеризующие качество используемой технологии и 
конкурентоспособность продукции. Например, рассматривая четыре уровня, имеем:

-уровень, не соответствующий требованиям рынков РФ;

-уровень, соответствующий требованиям рынков РФ;

-уровень, соответствующий требованиям мировых рынков;

-уровень, превышающий мировой.

Введенные виды продукции и уровни конкурентоспособности формально можно представить:

$$
\Pi_{i}(i=1 \div n) ; \quad Y_{e}(e=1 \div 4)
$$

В качестве входных параметров в моделях используется следующая информация: о затратах предприятия и их структуре, о рыночных показателях товаров, о налогах.

Для каждого из видов продукции задается себестоимость $(C)$ и ее структура $(S C)$, например:

$C_{n p}=M P+O T+A+\Pi P$,

$C_{\text {полн }}=C_{n p}+У P+K P$,

где $C_{n p}$ - производственная себестоимость,

$M P$ - материальные расходы,

$O T$ - расходы на оплату труда,

$A$ - суммы начисленной амортизации,

ПР - прочие расходы,

$C_{\text {полн }}-$ полная себестоимость,

УР - управленческие расходы,

$K P$ - коммерческие расходы.

В качестве рыночной информации (РИ) используются: по цене;

- коэффициент эластичности спроса товара

- коэффициент эластичности спроса товара по доходу потребителей;

- коэффициент изменения дохода потребителей;

- коэффициент эластичности спроса товара по цене взаимозаменяемого товара;

- индекс качества товара;

- рыночная цена товара;

- рыночная доля предприятия;

- прогноз изменения товарной массы, поставляемой конкурентами на рынок.

Информация о налогах (ИН) включает

a) налоги, оплачиваемые предприятием:

- суммарный налог в абсолютном выражении;

- налог с прибыли (\%);
- налог с выручки (\%);

- налог с валового дохода (\%);

б) налоги с доходов членов трудового коллектива:

- начисления на оплату труда (единый социальный налог) (\%);

- налог на доходы физических лиц (\%);

в) налоги, оплачиваемые потребителем:

- налог на добавленную стоимость (\%);

- акцизный налог (\%);

- таможенные платежи (\%) и другие возможные налоги в зависимости от особенностей предприятия-производителя и потребителей его продукции.

В подходе на основе расчета прибыли или индекса прибыли определяются для заданного интервала времени $T=T_{0} \div T_{c}$ в определенные моменты времени основные параметры деятельности предприятия - прибыль $\Pi P(t)$ или индекс прибыли $\overline{\Pi P}(t)[1,2]$.

Индекс прибыли предприятия определяется как отношение прибыли в момент времени $t$ к ее значению в предыдущий момент времени $t-1$.

$$
\overline{\Pi P}(t)=\frac{\Pi P(t)}{\Pi P(t-1)} .
$$

Прибыль от реализации продукции, работ, услуг базового и анализируемого периодов определяется по формулам:

$$
\begin{gathered}
\Pi P(t-1)=N(t-1)(Ц(t-1)-C(t-1)) \\
\Pi P(t)=N(t)(Ц(t)-C(t))
\end{gathered}
$$

где $N(t-1)$ и $N(t)$ - объем производства и реализации продукции, работ, услуг в натуральном выражении соответственно в базовом и анализируемом периодах;

$Ц(t-1)$ и $Ц(t)$ - цены реализации единицы продукции соответственно в базовом и анализируемом периодах;

$C(t-1)$ и $C(t)$ - себестоимость единицы продукции соответственно в базовом и анализируемом периодах.

Последовательно подставляя в формулу (1) выражения для определения параметрических показателей, формирующих прибыль предприятия, можно получить [1,2]:

$$
\overline{\Pi P}(t)=\frac{b(Ц(t)-C(t))}{C(t-1) \times\left(\frac{Ц(t-1)}{C(t-1)}-1\right)}=\frac{b\left(\frac{d Ц(t-1)}{C(t-1)}-\frac{C(t)}{C(t-1)}\right)}{p-1}=
$$




\begin{tabular}{|c|c|c|c|c|c|c|}
\hline Impact Factor: & $\begin{array}{l}\text { ISRA (India) } \\
\text { ISI (Dubai, UAE } \\
\text { GIF (Australia) } \\
\text { JIF }\end{array}$ & $\begin{array}{l}=1.344 \\
=0.829 \\
=0.564 \\
=1.500\end{array}$ & $\begin{array}{l}\text { SIS (USA) } \\
\text { PИНЦ (Russia) } \\
\text { ESJI (KZ) } \\
\text { SJIF (Morocco) }\end{array}$ & $\begin{array}{l}=0.912 \\
=0.234 \\
=1.042 \\
=2.031\end{array}$ & $\begin{array}{l}\text { ICV (Poland) } \\
\text { PIF (India) } \\
\text { IBI (India) }\end{array}$ & $\begin{array}{l}=6.630 \\
=1.940 \\
=4.260\end{array}$ \\
\hline
\end{tabular}

$$
=\frac{b\left(p d-\frac{K_{H} \times C_{\text {nep }}+\frac{C_{\text {nocm }}+\Delta C_{\text {nocm }}}{b \times N(t-1)}}{C(t-1)}\right)}{p-1}=\frac{b\left(p d-K_{H} \times r-\frac{C_{n o c m}(1+f)}{C(t-1) \times b \times N(t-1)}\right)}{p-1}=
$$

где $C_{n е р}-$ переменные затраты на единицу продукции базового периода;

$C_{\text {nост }}$ - условно-постоянные затраты в абсолютном выражении на выпуск продукции базового периода;

$\Delta C_{\text {пост }}$ - прирост условно-постоянных затрат в анализируемом периоде;

$$
\overline{\Pi P}(t)=\frac{b\left(p d-K_{H} \times r\right)-(1-r)(1+f)}{p-1}
$$

Параметрическая модель (5) была получена для предприятий, выпускающих продукцию одного наименования (одной номенклатурноассортиментной позиции) или многих наименований, но формирующих определенный комплект. Такие предприятия занимают незначительный удельный вес среди всех хозяйствующих субъектов, поэтому возникает вопрос о том, можно ли воспользоваться этой моделью для предприятий, выпускающих более одной номенклатурно-ассортиментной позиции продукции.

Для определения индекса прибыли предприятия при многономенклатурном производстве необходимо значения входящих в модель параметров вычислять по формулам, которые учитывают структурные сдвиги выпускаемой продукции при переходе из базового в анализируемый период $[1,2]$.

На основании полученных предприятием данных об объемах выпускаемой продукции $(V)$, о себестоимости (C) и ее структуре (SC), рыночной информации $(P И)$, информации о налогах $(И Н)$, для каждого из видов продукции $\left(\Pi_{i}\right)$ для настоящего момента времени $(t)$, входящего в период анализа предприятия ( $T=T_{o} \div T_{c}$ ), рассчитывается величина прибыли $(\Pi P(t))$ и индекс прибыли $(\overline{\Pi P}(t))$.

При расчете индекса прибыли в момент $t$ информация о прибыли $\Pi P(t-1)$ должна быть заданной.
$K_{u}-$ коэффициент изменения переменных затрат базового периода в анализируемом периоде.

В окончательном виде полученное выражение можно записать [1]:
Таким образом, общий алгоритм расчета индекса прибыли имеет вид:

задание $\left(V, C, S C, P И, И H, T=T_{o} \div T_{c}\right)$ $\rightarrow(t) \rightarrow \Pi P(t) \rightarrow$ задание $\Pi P(t-1) \rightarrow$ $\overline{\Pi P}(t)$.

Приведенное описание подхода на основе расчета прибыли и индекса прибыли к построению моделей предприятий с незначительно изменяющейся структурой будем в дальнейшем использовать для предприятий с изменяющейся структурой с учетом их специфических особенностей.

Если в процессе развития на интервале времени $\quad T=T_{o} \div T_{c}$ предприятие осуществляет кардинальные структурные изменения в отдельные периоды времени $t=(t-1) \div t$, выражающиеся, например, в освоении новых, прогрессивных и перспективных видов продукции $(H B)$, снятии с производства устаревшей и малоперспективной продукции $(\mathrm{CH})$, то данные особенности должны быть отражены в модели.

Расчеты индекса прибыли при структурных сдвигах следует производить по той же параметрической модели типа (5) с небольшой разницей, учитывающей особенности производства. Модификация параметрической модели индекса прибыли выглядит следующим образом [1]: 


\begin{tabular}{|c|c|c|c|c|c|c|}
\hline Impact Factor: & $\begin{array}{l}\text { ISRA (India) } \\
\text { ISI (Dubai, UAE } \\
\text { GIF (Australia) } \\
\text { JIF }\end{array}$ & $\begin{array}{l}=1.344 \\
=0.829 \\
=0.564 \\
=1.500\end{array}$ & $\begin{array}{l}\text { SIS (USA) } \\
\text { PUHЦ (Russia) } \\
\text { ESJI (KZ) } \\
\text { SJIF (Morocco) }\end{array}$ & $\begin{array}{l}=0.912 \\
=0.234 \\
=1.042 \\
=\mathbf{2 . 0 3 1}\end{array}$ & $\begin{array}{l}\text { ICV (Poland) } \\
\text { PIF (India) } \\
\text { IBI (India) }\end{array}$ & $\begin{array}{l}=6.630 \\
=1.940 \\
=4.260\end{array}$ \\
\hline
\end{tabular}

$$
\overline{\Pi P}(t)=\frac{b\left(p_{c} d-K_{H} \times r\right)-(1-r)(1+f)}{p-1}
$$

где $p_{c}-$ коэффициент рентабельности производства, учитывающий структурные сдвиги.

$$
p_{c}=\frac{\sum_{m+l}^{m+n+l} N(t)_{i} Ц(t-1)_{i}}{\sum_{m+l}^{m+n+l} N(t)_{i} C(t-1)} .
$$

В данном случае в подходе, основанном на расчете прибыли, индекса прибыли, на интервалах времени $t=(t-1) \div t \quad$ для каждого нового осваиваемого вида продукции $(H B)$ рассчитывается величина ее себестоимости $\left(C_{H B}\right)$, структура себестоимости $\left(S C_{H B}\right)$, задается рыночная информация $\left(Р И_{H B}\right)$, информация о налогах $\left(И H_{H B}\right)$.

При расчете значений прибыли, индекса прибыли дополнительно вводятся математические комплексы, соответствующие новым видам продукции $(H B)$ и исключаются комплексы, относящиеся к продукции, снимаемой с производства $(\mathrm{CH})$.

Рассмотренный подход к определению прибыли, индекса прибыли используется для приближенной экспресс-оценки состояния предприятий с учетом их специфических особенностей. Он особенно важен для тех предприятий, где прибыль и ее динамика являются определяющими параметрами развития.

Вместе с тем, в данном подходе не учитываются важнейшие особенности, влияющие на функционирование и развитие предприятий:

-влияние технологий на объем и качество выпускаемой продукции;

-влияние числа и квалификации специалистов на объем и качество продукции;

-влияние инвестиций на развитие основных фондов, объем выпускаемой продукции.

Наряду с вышеизложенным подходом на основе расчета прибыли и индекса прибыли, в работе предлагается системный подход анализа состояния предприятия, учитывающий взаимодействие производственных процессов, инновационных технологий с финансовоэкономическим структурами предприятия [4].

В данной модели с помощью трехфакторной производственной функции учитывается зависимость объема выпускаемой продукции от численности работающих на предприятии, уровня используемых в производстве технологий, объема производственных фондов, который, в свою очередь, зависит от объема инвестиций [2,3]:

$$
\begin{aligned}
& X^{t}=F_{x}^{t}\left(L_{x}^{t}, T_{x}^{t}, \Phi_{x}^{t}\right) ; \\
& \Phi_{x}^{t}=F_{\phi}^{t}\left(I_{x}^{t}\right),
\end{aligned}
$$

где $X^{t}$ - объемы выпуска товаров и рыночных услуг в постоянных основных ценах;

$L_{x}^{t} \quad-$ численность работающих на предприятии;

$T_{x}^{t}-$ уровень используемых в производстве технологий;

$\Phi_{x}^{t}$ - объем основных производственных фондов;

$I_{x}^{t}$ - объем инвестиций.

Также могут быть заданы производственные функции для каждого из $n$ видов продукции, выпускаемой на предприятии:

$$
\begin{gathered}
X_{1}^{t}=F_{x 1}^{t}\left(L_{x 1}^{t}, T_{x 1}^{t}, \Phi_{x 1}^{t}\right) \\
\Phi_{x 1}^{t}=F_{\phi 1}^{t}\left(I_{x 1}^{t}\right) \\
X_{2}^{t}=F_{x 2}^{t}\left(L_{x 2}^{t}, T_{x 2}^{t}, \Phi_{x 2}^{t}\right), \\
\Phi_{x 2}^{t}=F_{\phi 2}^{t}\left(I_{x 2}^{t}\right)
\end{gathered}
$$

$X_{n}{ }^{t}=F_{m}^{t}\left(L_{m}^{t}, T_{m}^{t}, \Phi_{m}^{t}\right), \Phi_{m}^{t}=F_{\phi n}^{t}\left(I_{m}^{t}\right)$

При реализации предлагаемого подхода должны быть найдены функции $F_{x}^{t}, F^{t}$, далее вычисляются другие показатели деятельности предприятия, в том числе прибыль.

Для нахождения функций, характеризующих изменение объема выпускаемой продукции $\left(F_{x}^{t}\right)$, используются методы идентификации [2,3], при этом задается информация о параметрах $X^{i}, L_{x}^{i}$, $T_{x}^{i}, \Phi_{x}^{i}$ в предыдущие моменты времени $\left(t<t_{i}\right)$.

Динамика основных фондов определяется их начальным объемом, вводом и выбытием:

$$
\Phi_{x}^{t+1}=\left(1-r_{x}^{t}\right) \Phi_{x}^{t}+\Delta \Phi_{x}^{t},
$$

где $\quad r_{x}^{t}-$ коэффициент выбытия основных фондов;

$\Delta \Phi_{x}^{t}$ - ввод фондов в текущем периоде.

Ввод фондов определяется освоенными инвестициями предыдущего периода:

$$
\Delta \Phi_{x}^{t}=I_{x}^{t-1}
$$

Общий объем инвестиций обеспечивается собственными возможностями предприятия $\left(I S_{x}^{t}\right)$, расходами на инвестиции регионального бюджета $\left(I R_{x}^{t}\right)$, федерального бюджета $\left(I F_{x}^{t}\right)$, заемными средствами $\left(I Z_{x}^{t}\right)$ :

$$
I_{x}^{t}=I S_{x}^{t}+I R_{x}^{t}+I F_{x}^{t}+I Z_{x}^{t} .
$$


Основным финансовым результатом является прибыль (убыток) от реализации товаров и услуг:

$$
\begin{aligned}
& P R_{x}^{t}=P_{x}^{t} X^{t}\left(1-m_{x}^{t}\right)-a_{x} \Phi^{t}\left(1-m_{x}^{t}\right)- \\
& -a_{x} \Phi_{x}^{t}-z_{x}^{t} L_{x}^{t}\left(1+n_{l}^{t}\right) .
\end{aligned}
$$

Параметры уравнения прибыли имеют следующий смысл:

$P_{x}^{t}$ - индекс роста основных цен;

$m_{x}^{t}$ - удельные материальные затраты;

$a_{x}$ - норма амортизации основных фондов;

$z_{x}^{t}-$ средняя заработная плата;

$n_{l}^{t}-$ начисления на заработную плату.

Уравнение распределения прибыли включает налоги на прибыль $\left(N I_{x}^{t}\right)$, расходы на выплату дивидендов (для акционерных обществ) $\left(D P_{x}^{t}\right)$, на инвестиции в основные фонды для производства товаров и рыночных услуг (собственные инвестиции предприятия) $\left(I S_{x}^{t}\right)$, на создание фондов накопления на предприятии $\left(I F^{t}\right)$ :

$$
P R_{x}^{t}=\underset{\text { В }}{N I_{x}^{t}+D P_{x}^{t}}+\underset{\text { простейшем }}{I S_{x}^{t}+I F_{x}^{t}} \underset{\text { случае }}{\text { политику }}
$$
распределения прибыли можно определить структурными управляющими параметрами:

$$
\begin{aligned}
& D P_{x}^{t}=\alpha_{p} P R_{x}^{t} ; I S_{x}^{t}=\beta_{p} P R_{x}^{t} ; I F_{x}^{t}= \\
& =\gamma_{p} P R_{x}^{t} .
\end{aligned}
$$

Таким образом, для построения и использования модели предприятия с незначительно изменяемой структурой на основе системного подхода необходимо:

- задание для каждого из $n$ видов выпускаемой продукции объемов используемых инвестиций $\left(I_{x}^{t}\right)$ и расчет стоимостей основных производственных фондов $\left(\Phi_{x}^{t}\right)$;

- определение численности работающих предприятия $\left(L_{x}^{t}\right)$, параметров технологических процессов $\left(T_{x}^{t}\right)$, задействованных в процессе выпуска каждого вида продукции;

- выполнение математической процедуры по расчету функций $F_{x}^{t}, F_{\phi}^{t}$;

- вычисление объема $\left(X^{t}\right)$ производимой на предприятии продукции;

- расчет затрат на выпуск продукции;

- задание рыночной информации (РИ) и информации о налогах $(И Н)$;

- расчет основных абсолютных и относительных показателей, характеризующих деятельность предприятия (прибыль, индекс прибыли, рентабельность и т.д.).

В данном подходе специфика предприятий учитывается как путем выделения $n$ видов продукции, так и описанием применяемых на предприятиях технологий $\left(T_{x}^{t}\right)$.
Приведенные подходы к построению моделей предприятий с незначительно изменяющейся структурой будем в дальнейшем использовать для предприятий с изменяющейся структурой с учетом их специфических особенностей.

Если в процессе развития на интервале времени $\quad T=T_{o} \div T_{c}$ предприятие осуществляет кардинальные структурные изменения в отдельные периоды времени $t=(t-1) \div t$, выражающиеся в освоении новых видов продукции $(H B)$, снятии с производства продукции $(\mathrm{CH})$, существенно изменяет технологию $(T)$, обеспечивает рост численности работников предприятия $(L)$, то данные особенности должны быть отражены в модели.

При построении модели предприятия на основе системного учета инвестиций, уровня технологий, численности работающих, объема основных производственных фондов для случая кардинальных структурных изменений сохраняются основные процедуры, введенные для предприятий с малоизменяющейся структурой, но при этом задается дополнительная информация о новых видах продукции $(H B)$ и исключается для снимаемых с производства $(\mathrm{CH})$.

Вместе с тем, освоение новых видов продукции, особенно в случае существенного изменения ее качества, конкурентоспособности и связанным с этим внедрением новых технологий, привлечением дополнительного числа высококвалифицированных специалистов приводит к необходимости замены существующих на предприятии методов оценки качества специалистов, технологических процессов, методов реализации процедур по расчету функций $F_{x}^{t}, F^{t}{ }_{\phi}$. [5]

Для анализа и планирования производства и распределения продукции отдельного предприятия можно применить межпродуктовый балансовый метод [2,3].

Основу баланса составляет совокупность всех продуктов, производимых анализируемым предприятием. Всю производимую предприятием продукцию можно разделить на промежуточную и конечную. Промежуточной называется та часть продукции, которая идет в дальнейшую переработку в пределах предприятия и образует текущие материальные затраты. Конечной называют ту продукцию, которая окончательно покидает пределы производства и используется для реализации. Одна и та же продукция на предприятии может делиться на промежуточную и конечную, т.е. часть продукции используется как средства производства на предприятии, часть идет на продажу $[6,7]$. 
Каждый продукт дважды фигурирует в балансе: рассматривается его производство и потребление. Производству продукции соответствует определенная строка, потреблению продукции - определенный столбец. Обозначим через $i$ номер производимой продукции, через $j-$ потребляемой продукции (промежуточной продукции). Величины $x_{i j}$ показывают стоимость средств производства (стоимость $i$-й продукции), которые необходимо затратить для создания $j$-й продукции.

В столбцах баланса отражается структура материальных затрат $\left(x_{1 j}, x_{2 j}, \ldots x_{8 j}\right)$ и чистой $j$-й продукции $\left(v_{j}+m_{j}\right)$, где $v$ - оплата труда, $m-$ чистый доход.

Сумма материальных затрат и чистой продукции равна валовой $j$-й продукции:

$$
X_{j}=\sum_{i=1}^{n} x_{i j}+v_{j}+m_{j}
$$

Формула (17) охватывает систему из $n$ уравнений, отражающих стоимостной состав промежуточной продукции, которая идет в дальнейшую переработку в пределах анализируемого предприятия. В строках межпродуктового баланса содержаться данные о распределении объема производимой продукции.

Предложенная в статье система моделей может использоваться для оценки состояния предприятий регионов (субъектов РФ), имеющих структурные подразделения, обеспечивающие транспортные услуги, эксплуатацию, ремонт автомобилей, а также на отдельных предприятия, занимающихся только автосервисом [8-10].

\section{References:}

1. Bogatin YV (1998) Ocenka ehffektivnosti proizvodstvennogo biznesa i investicij. Monografiya. - Rostov-n/D, RGEHA, 1998. $272 \mathrm{p}$.

2. Potopahin VA, Potopahina MV (2004) Dinamicheskie modeli i tekhnologii upravleniya $\mathrm{v}$ razvitii mnogourovnevyh sistem. - Moscow: Vuzovskaya kniga, $2004-400$ p.

3. Dudchak VV, Potopahin VA (2005) Adaptivnoe upravlenie predpriyatiyami oboronno-promyshlennogo kompleksa.

Moscow: Vuzovskaya kniga, 2005 - 300 p.

4. Markov OD (1999) Avtoservis: Rynok, avtomobil', klient. - Moscow: Transport, 1999.

5. Karablin OV (2010) Jekonomika i tehnologija proizvodstva sistem i oborudovanija kompleksov/O.V. Karablin,A.S. Reshenkin. Rostov n/D, 2010.

6. Vorob'ev SS, Vorob'ev SA (2011) Proektirovanie processa okazaniya uslug. Metodicheskoe posobie, RIO RTIST YURGUEHS, 2011, 15p.

7. Vorob'ev SA, Vorob'ev SS (2012) Tekhnicheskoe obsluzhivanie i remont avtomobilej Uchebnoe posobie, RIO RTIST FGBOU VPO «YURGUEHS», 2012, 127 p.

8. Reshenkin AS, Vorob'ev SS, Andros DS, Babkin VG, Ganchurin AY (2015) Logistizaciya predpriyatij pochtovoj svyazi na osnove modeli razgranicheniya zon obsluzhivaniya. Theoretical \& Applied Science. 2015. № 11. pp. 161-170.

9. Reshenkin AS, Vorob'ev SS, Aslanyan RN, Sattarov TK, Antipanov MO (2015) Statistisheskaya model' prongoza pochtovyh gruzopotokov, osnovannaya na principah logistiki Theoretical \& Applied Science. 2015. № 12. pp. 28-33.

10. Vorob'ev SS, Vorob'ev SA (2014) Vvedenie v professional'nuju dejatel'nost'. (servis transportnyh sredstv) uchebnoe posobie / Pechataetsja po resheniju redakcionnoizdatel'skogo soveta Donskogo gosudarstvennogo tehnicheskogo universiteta. Rostov-na-Donu, 2014. 